University of Wollongong

Research Online

Faculty of Law, Humanities and the Arts Papers (Archive)

Faculty of Arts, Social Sciences \& Humanities

$1-1-2019$

\title{
Enactive social cognition: Diachronic constitution \& coupled anticipation
}

Alan Jurgens

University of Wollongong, awj949@uowmail.edu.au

Michael D. Kirchhoff

University of Wollongong, kirchhof@uow.edu.au

Follow this and additional works at: https://ro.uow.edu.au/lhapapers

Part of the Arts and Humanities Commons, and the Law Commons

Research Online is the open access institutional repository for the University of Wollongong. For further information contact the UOW Library: research-pubs@uow.edu.au 


\title{
Enactive social cognition: Diachronic constitution \& coupled anticipation
}

\begin{abstract}
This paper targets the constitutive basis of social cognition. It begins by describing the traditional and still dominant cognitivist view. Cognitivism assumes internalism about the realisers of social cognition; thus, the embodied and embedded elements of intersubjective engagement are ruled out from playing anything but a basic causal role in an account of social cognition. It then goes on to advance and clarify an alternative to the cognitivist view; namely, an enactive account of social cognition. It does so first by articulating a diachronic constitutive account for how embodied engagement can play a constitutive role in social cognition. It then proceeds to consider an objection; the causal-constitutive fallacy (Adams \& Aizawa, 2001, 2008; Block, 2005) against enactive social cognition. The paper proceeds to deflate this objection by establishing that the distinction between constitution and causation is not co-extensive with the distinction between internal constitutive elements and external causal elements. It is then shown that there is a different reason for thinking that an enactive account of social cognition is problematic. We call this objection the 'poverty of the interactional stimulus argument'. This objection turns on the role and characteristics of anticipation in enactive social cognition. It argues that anticipatory processes are mediated by an internally realised model or tacit theory (Carruthers, 2015; Seth, 2015). The final part of this paper dissolves this objection by arguing that it is possible to cast anticipatory processes as orchestrated as well as maintained by sensorimotor couplings between individuals in face-to-face interaction.
\end{abstract}

\section{Disciplines}

Arts and Humanities | Law

\section{Publication Details}

Jurgens, A. \& Kirchhoff, M. D. (2019). Enactive social cognition: Diachronic constitution \& coupled anticipation. Consciousness and Cognition, 70 1-10. 
Title: Enactive Social Cognition: Diachronic Constitution \& Coupled Anticipation

\title{
Authors:
}

Alan Jurgens (jurgens.alan@gmail.com)

Michael D. Kirchhoff (kirchhof@uow.edu.au)

\section{Affiliations:}

1. Department of Philosophy, Faculty of Law, Humanities and the Arts, University of Wollongong, Wollongong, Australia.

2. Department of Philosophy, Faculty of Law, Humanities and the Arts, University of Wollongong, Wollongong, Australia.

Acknowledgements: Kirchhoff was supported by an Australian Research Council Discovery Project "Minds in Skilled Performance" (DP170102987). Jurgens was funded by a University Postgraduate Award and an International Postgraduate Tuition Award from the University of Wollongong.

\begin{abstract}
This paper targets the constitutive basis of social cognition. It begins by describing the traditional and still dominant cognitivist view. Cognitivism assumes internalism about the realisers of social cognition; thus, the embodied and embedded elements of intersubjective engagement are ruled out from playing anything but a basic causal role in an account of social cognition. It then goes on to advance and clarify an alternative to the cognitivist view; namely, an enactive account of social cognition. It does so first by articulating a diachronic constitutive account for how embodied engagement can play a constitutive role in social cognition. It then proceeds to consider an objection; the causal-constitutive fallacy (Adams \& Aizawa 2001, 2008; Block 2005) against enactive social cognition. The paper proceeds to deflate this objection by establishing that the distinction between constitution and causation is not coextensive with the distinction between internal constitutive elements and external causal elements. It is then shown that there is a different reason for thinking that an enactive account of social cognition is problematic. We call this objection the 'poverty of the interactional stimulus argument'. This objection turns on the role and characteristics of anticipation in enactive social cognition. It argues that anticipatory processes are mediated by an internally realised model or tacit theory (Carruthers 2015; Seth 2015). The final part of this paper dissolves this objection by arguing that it is possible to cast anticipatory processes as orchestrated as well as maintained by sensorimotor couplings between individuals in face-to-face interaction.
\end{abstract}

Keywords: Social cognition; enactivism; intersubjectivity; diachronic constitution; theory of mind; sensorimotor contingencies; causal-constitutive fallacy. 


\section{Introduction}

There is still substantial dispute about the boundaries of social cognition, and what determines drawing the boundaries in one way as opposed to a different way. Is social cognition wholly and exhaustively constituted by elements in the brain or is it, rather, constituted in ongoing dynamic and interactive engagement between agents?

Social cognition has often been, and still is, presented as the inevitable result of the following key cognitivist assumptions: (i) realiser internalism, which states that cognition, and by extension social cognition, is realised by processes in the head of individuals; and (ii) methodological internalism, which states that the proper unit of social cognitive analysis should be the individual agent (Carruthers 2015; Herschbach 2012; Schönherr \& Westra 2017). The second assumption is a commitment to the idea that perception even if embedded in and scaffolded by sociocultural practices needs to be informed by conceptual knowledge (Carruthers 2015; Schönherr 2016; Schönherr \& Westra 2017). What fuels this assumption is the observation that there is no one-to-one mapping between mental states and behavior for "actions and facial expressions that manifest any given mental state are always contextsensitive, and vary depending on the agent's other mental states and circumstances" (Carruthers 2015, p. 499). Cognitivism thus posits the existence of an inner model or tacit theory, comprised of rules and representations, to explain the nature of social cognition. ${ }^{1}$

In this paper, we aim to both clarify and further develop an enactivist alternative to cognitivist social cognition. Enactivism, especially in its radical formulation (Hutto \& Myin 2013), casts the vast majority of cognitive activity as constituted in embodied and situated activity. Applying the enactivist framework to social cognition thus frontloads the central importance of embodied and social interaction in explaining the vast sea of social cognition. ${ }^{2}$

Defenders of cognitivist social cognition can, and do, acknowledge that embodied activity and social interaction plays a role in social cognition. Thus, simply touching on interaction as contributing to social cognition is uncontroversial. ${ }^{3}$ However, advocates of enactivism claim that

\footnotetext{
${ }^{1}$ We do not consider simulation theory in this paper, but focus our attention on the dominant framework of theory theory. It is worth noting however that even simulationist accounts of social cognition such as Gallese's (2014) embodied simulation account is open to the same theory theory objections considered here because these accounts could be argued to only get a grip on social cognition in virtue of being informed by a tacit theory.

2 The enactive framework we propose does not rule out that some non-interactive, offline forms of social cognition might involve neurally realised representations or conceptual knowledge. For example, when thinking about having a difficult conversation with a partner one might rehearse the beginning of the conversation in one's head to anticipate the possible reactions of one's partner, which might involve representations and conceptual knowledge. Moreover, we see no reason to deny that social cognition is multi-dimensional and can be cast on a continuum. For instance, we will argue that infant-caretaker dyads illustrate cases of extended emotion regulation. This does not hold in all cases of social cognition. For example, one might only be able to manifest certain states of euphoria when participating in certain kinds of crowd behavior - this would be an example of Wilson's (2004) social manifestation thesis. Yet granting the latter does not rule out cases such as the former.

${ }^{3}$ Overgaard and Michael (2015) raise a two-horned dilemma for enactive social cognition on this precise point. Either "social cognition, quite generally, is wholly a matter of processes outside the individual" (2015, p. 175). Or, social cognition must not be reduced solely to what is going on inside an individual as action and interaction play important, even if only, causal roles. This induced dilemma leads Overgaard
} 
those in the grip of cognitivism only pay lip service to interaction in social cognition. In this paper we shall defend the enactive view that social cognition is constituted in interaction, thus alleviating the need, always and necessarily, to appeal to social cognition as mediated and grounded in the dynamics of internal, brain-based models.

We start by articulating the enactivist position that interpersonal interaction is constitutive of social cognition. We do this by offering a diachronic characterisation of the notion of constitution as it underlies enactivist views of social cognition (Kirchhoff 2015; cf. Gallagher 2018). Crucially, we show that: (i) both the explanandum and explanans of social cognition are processes, and thus ineliminably temporal; (ii) the relation between microscale (local) and macroscale (global) processes cannot hold wholly and exclusively at a synchronic instant $t$; (iii) the local and global processes stand in a relation of circular causation, as per the slaving principle in physics; and (iv) that social cognition is a novel, macroscale process that cannot be reductively explained by reference to processes residing and operating at the microscale simpliciter. This lends further support to enactivist proposals about the diachronic nature of social cognition (De Jaegher et al. 2010; Froese \& Gallagher 2012; Gallagher 2018).

We then turn to consider an objection to our account of diachronic constitution; namely, the causal-constitutive fallacy (Adams \& Aizawa 2001, 2006, 2008; Aizawa 2010; Block 2005; Carruthers 2015; Herschbach 2012). The fallacy states that any claim about the extended nature of cognition that starts from observations about causal coupling unjustifiably infers facts about constitution from facts about causation. Hence, our diachronic account of constitution allegedly falls prey to this kind of worry. Following Hurley (2010) we show that this objection can be questioned, for it helps itself to the question-begging assumption that the distinction between constitution and causation is co-extensive with a distinction between internal constitutive elements and external causal elements. We argue, with Hurley, that without further evidence the causal-constitutive fallacy is itself a fallacy (for additional discussion, see also Abramova \& Slors 2018; Kirchhoff 2015, 2017).

Even if we can diffuse the causal-constitutive fallacy, there is a different reason for being skeptical about enactive social cognition. We call this the 'poverty of the interactional stimulus' argument (Carruthers 2015). It turns on the role and characteristics of anticipation in social cognition. It can be put as follows: there is a substantial problem with the claim that social cognition is constituted in interaction, for moment-by-moment interactional stimuli are too informationally impoverished to allow any individual to make sense of the richness and variety of behaviors that another agent might perform at any given moment. It is not possible to anticipate why some agent does this or that without presupposing that agents possess some kind of tacit theory of social cognition. Thus, Carruthers (2015, p. 499) claims that "enactivism cannot obviate the need for tacit theory" in its explanation of social cognition. Our response to this objection builds on the distinction between anticipation and situated interaction. The poverty of the interactional stimulus argument turns on the assumption that knowledge of social cognition

and Michael to conclude that the enactive position is either implausible (first horn) or trivial (second horn). Enactivists however accept that internal brain processes play a constitutive role in facilitating social cognition. So the first horn is not a plausible argument against enactive social cognition. Yet enactivists also go further by embedding neural dynamics in an extended and constitutive nexus comprising embodied and situated dynamics in accounting for social cognition. So the second horn is not plausible, if cast as an argument against enactive social cognition. Enactive social cognition lies between these strong and weak versions that Overgaard and Michael (2015) attribute to it. 
underlies and therefore enables the ability to engage in multi-agent interaction. We flip this picture on its head by showing that anticipatory processes can, when in the right kind of circumstances, be partially constituted by online interaction between agents. This allows us to purge the enactivist appeal to counterfactual anticipation of sensorimotor dependencies from any unnecessary association with cognitivism (i.e., the appeal to tacit theory and representation as the basis of social cognition), on the one hand, and internalism, on the other. We argue that the enactive appeal to anticipatory processes that target sensorimotor dependencies may, in the right circumstances, be constituted in the dynamic coupling between individuals in situated action. We conclude that even if social cognition rests on agentive abilities to anticipate counterfactual relations between perception and action (cf. Di Paolo et al. 2017; Noë 2004, 2009; Seth 2015), these abilities are grounded in situated, ongoing engagement with other agents, over multiple spatial and temporal scales. ${ }^{4}$

\section{Enactive Social Cognition}

In this section our agenda is to establish the constitutive claim of enactive social cognition. We start, section 2.1., with a neutral description of a paradigmatic example of face-to-face social cognition; namely, emotion regulation in infant/caretaker dyads. We then turn to address, section 2.2., the enactive claim that the interactions themselves are at least partly constitutive of social cognition. We show that to make sense of this enactive claim one must turn away from the standard synchronic conception of constitution and adopt a notion of constitution that is dynamic and diachronic. The state of the art in enactive social cognition however bifurcates when addressing how best to understand the notion of diachronic constitution. Some argue that this is best explained through the lens of the new mechanist framework (Abramova \& Slors 2018; Gallagher 2018). Others pursue an explanation in non-mechanistic terms, cast via the framework of dynamical systems theory (Di Paolo \& De Jaegher 2017; De Jaegher 2018). We propose to chart a course between the options of new mechanism, on the one hand, and nonmechanism, on the other. Rather than defending one or the other, our articulation of diachronic constitution is applicable to both friend and foe of the mechanistic framework. ${ }^{5}$

\subsection{Case Study: Infant-Caretaker Interaction}

We take it to be nearly if not entirely uncontroversial to say that the basic form of human social cognition takes place in face-to-face interaction with others (Krueger 2010). One of the earliest

\footnotetext{
${ }^{4}$ This is not meant to remove the importance of internal processes. Our point is rather that anticipation can sometimes be realised or constituted by the dynamics of entire extended systems, including brains, eyes, hands, and so on, such that what enables anticipation in the first place has to do with situated and ongoing activity, given that it is the latter coupling agent to environment (or another agent) via perception and action (see also Gallagher 2017).

${ }^{5}$ We take this to be a virtue of our proposal, for it avoids getting stuck in the long-grass having to defend either mechanistic or non-mechanistic schemes or some hybrid of both to articulate the constitution claim of enactive social cognition. Moreover, our proposal not only applies to enactive social cognition but more generally to any view of cognition that posits the relata of the constitution relation as being dynamic and processual. This is a real explanatory virtue of our constitutive proposal. Nevertheless, we cannot hope to address this in more detail in this paper - this will be a task for another occasion.
} 
examples of such interaction is the infant/caretaker dyad, where body posture, expressive gesture, vocalisation, gaze following, and so on, all play a role in the ongoing and recurrent engagement between infant and caretaker. The bedrock for this kind of face-to-face interaction can even be traced to shortly after birth, where infants have been shown to have a preference for engaging with faces of others, lending credibility to the view that the ability to engage in social forms of cognition is present very early in ontogeny. ${ }^{6}$ In infant/caretaker dyads, the infant recognises when she is being addressed by the caretaker and responds accordingly to the caretaker's playful or more serious emotions, conditioned on the caretaker's facial and vocal postures and gestures. The core features of this example are the infant recognising, attending to, and responding to the caretaker's interaction with the infant, and the reciprocal behaviour of the caretaker recognising, attending to, and responding to the infant's behaviour and interaction with herself. ${ }^{7}$

The so-called 'still face' experiment brings to light the importance of ongoing and synchronous engagement, and what happens when the generalised synchrony of nonverbal behaviors of the infant and caretaker breaks down (Varga 2015). In still face experiments, the infant is first engaged by her mother in a normal face-to-face interaction. This is followed by a period where the mother assumes a neutral facial expression, remaining motionless with a 'still face', which is then followed by the mother re-engaging the infant in normal face-to-face interaction (Gopnik \& Meltzoff 1996, p. 131). In these experiments, it has been shown that infants between 3 and 6 months become noticeably discouraged and upset during the second neutral face period, where synchronous, mutual interaction has broken down (Tronick et. al., 1978; Nagy et al., 2017). During this period the "infants withdraw from the interaction, avert their gaze, display negative affect, become increasingly distressed, start crying, and smile less" than during the normal engaged behaviour prior (Nagy et al., 2017, p. 2). Additionally, there is a noticeable spill-over effect after the mother re-engages with the infant. In this re-engagement phase, the infant will continue to avert its gaze from the mother, displays distress and in general, it will not re-engage with its mother to the same level as before the still phase (Nagy et al., 2017, p. 2).

We intend this example to highlight the variables that one can observe or at least reasonably approximate given the occurrent behavior. Cognitivists and enactivist are divided in how to explain what does the constitutive, as opposed to merely causal, work in cases such as infant/caretaker engagements. Enactive accounts stress that social cognition is constituted in a non-trivial extended process such that social cognitive processes are instantiated not in a single individual but in the coupling between individuals (Varga 2015). ${ }^{8}$ Coupling relations like these

\footnotetext{
${ }^{6}$ How to understand and explain this preference for faces is still under debate - see Barrett (2011, p. 2832) for discussion.

${ }^{7}$ We do not claim that this example speaks to sophisticated forms of social understanding. Still, we do not think this should count against it qualifying as a case of social cognition. Furthermore, even though the case we consider involves emotion regulation, this does speak against it being a form of social cognition, as there are strong reasons suggesting that affect and cognition are intimately linked and integrated (Colombetti \& Krueger 2015).

${ }^{8}$ We're working with the notion of extended in the active externalism sense of Clark and Chalmers (1998/ 2010), as opposed to the passive externalism of Putnam (1975) and Burge (1979). As in the classic Otto example by Clark and Chalmers (1998/2010), what is claimed to be extended is the cognitive process, not the subjects. In the Otto example, it is his process of remembering that is extended through the use of the notebook, not Otto himself nor his notebook. If the notebook is removed, this would cause a
} 
are known as generalised synchrony - the process whereby multiple systems or agents are driven to assemble into a single coherent ensemble. The cognitivist framework that we target states that even if individual agents showcase dynamic coupling in face-to-face interaction, such coupling is meaningless unless supplemented by an implicit grip of a folk psychological theory (Carruthers 2015). In the next section we will unpack how to think about the notion of constitution, when applied to enactive social cognition.

\subsection{Enactive Social Cognition and Diachronic Constitution}

Consider the following: you can leave your record player in the garage, return many years after and start using it again. But "if you accidentally leave your hamster in the loft, you will not have a hamster for very long." (Dupre \& Nicholson 2018, p. 15) This is obvious enough. Yet it speaks to a key theme of enactive social cognition; namely, action. Individual agents are always in need of acting in their environment to continue to exist. Such is their existential predicament. Action is at the root of what it is to be alive (Di Paolo 2009; Friston 2013). In social cognition, it is interaction that is at the foundation (De Jaegher et al. 2010).

The example above highlights a division between material objects (e.g., record players) and processes (e.g., remaining alive). Interaction is processual, through and through. Crucially, the distinction between material objects and processes maps onto two different conceptions of the constitution relation: synchronic and diachronic constitution. Only diachronic constitution is applicable to enactive social cognition (Gallagher 2018; Kirchhoff 2015). ${ }^{9}$

Synchronic constitution is the standard conception of how to think of constitution. In metaphysics, synchronic constitution is usually referred to as material constitution. However, the specification of constitution as a synchronic relation is also associated with related dependence relations such as realisation, composition and supervenience (Bennett 2011). The synchronic constitution relation can be framed in terms of how to fill out the following schema: a piece of marble, Piece, constitutes a specific statue, David, at a synchronic instant $t$ if and only if ? (Wasserman 2004, p. 694). It is widely agreed that a necessary condition for Piece to constitute David is that the constitution relation that holds between Piece and David involves two coincidence conditions. First, constitution requires spatial coincidence: Piece constitutes David at a synchronic instant $t$ only if Piece and David have the same spatial location at $t$. Second, constitution requires material coincidence: Piece constitutes David at a synchronic instant $t$ only if Piece and David share all the same material parts at $t$ (Wilson 2007, p. 5). Constitution is also understood to be asymmetric and a relation of relative fundamentality. Asymmetry means that if Piece constitutes David, then David does not, at the same time, constitute Piece. Relative fundamentality refers to the view that Piece is in some sense more

corresponding drop in Otto's behavioural competence in the same way that removing certain internal parts of the brain would (Clark \& Chalmers 1998/2010, p. 29). Similarly, in the infant/caretaker dyad it is the infant and the caretaker's social cognitive processes that are claimed to be extended, not the subjects themselves. See also Kirchhoff (2015) for a similar example of transactive memory where the process of remembering is extended between two agents working together to recall a specific experience.

${ }^{9}$ We take our conception of diachronic constitution to be a species of causation, i.e., constitutive causation, where constitutive causation is unidirectional. We capture this unidirectional feature in the terminology of continuous reciprocal causation. For further details on this issue, see Kirchhoff (2017). 
ontologically significant than David - viz., the parts are at a more fundamental ontological level than the whole.

By comparison, consider the Mexican wave as an example of social engagement. A Mexican wave is a common phenomenon in sports and occurs when individuals stand up slightly after the person next to them does, and so on, resulting in what appears to be a wave running or rolling through the crowd. There are several things to note about this example, all of which speak to adopting a notion of constitution cast in diachronic terms (see table 1 for an overview of the properties of synchronic and diachronic constitution).

First, a Mexican wave is an ontologically nested and multilayered process, as it is organised hierarchically into microscale (local) and macroscale (global) dynamics. Crucially, these differences in scale corresponds to a difference in the timescale over which local and global dynamics unfold - macroscale processes (i.e., the wave as it unfolds over space and time) exist over a longer timescale than microscale events or processes (i.e., individual agents standing up and down). Specifically, the Mexican wave loses and gains constituents at each moment of its existence and over its career. This observation sits in stark contrast with synchronic constitution, given the commitment of synchronic constitution to both spatial and material coincidence.

Second, in contrast to David/Piece, where the constitution relation holds between $\mathrm{X}$ and $Y$ at an instant $t$, the relation between local and global dynamics in the Mexican wave cannot hold exclusively at such a snapshot moment in time. On the one hand, coordination is required for this phenomenon to arise. This means that there must be a systematic or non-accidental correlation in the activity of the individual constituents, which, over multiple temporal and spatial scales make up the Mexican wave. On the other hand, the relation between the microscale and macroscale dynamics is time-dependent, in the specific sense that the dynamics at $t 1$ are partly constitutive of the configuration of the wave at $t 2$. So, temporally prior microscale dynamics feed into the configuration of the microscale and macroscale dynamics at temporally later stages.

Third, the formation of microscale dynamics, i.e., people standing up and sitting down, give rise to a macroscale and relatively stable pattern, which 'enslaves' the behaviour of individual agents by instantiating a normative standard for behaviour. On the one hand, this highlights that engagement is a necessary component in the constitution of the Mexican wave. Following De Jaegher et al. 2010, we take engagement to specify a specific social interaction starting to acquire a momentum of its own, as the ongoing roll through the crowd sweeps up others as it moves along. So the Mexican wave is a case of complex social engagement that involves a raft of different participants, imposing temporal demands on one another. On the other hand, once established, the claim that the wave 'sweeps up others as it moves along' highlights that the activity of standing up and sitting down at the local scale is driven and shaped by the Mexican wave in virtue of it being a shared practice. This means that the cultural practice of engaging in a Mexican wave, at the global scale, combined with the dynamics of the individuals at the microscale "can be seen as elements of a single adaptive dynamical system" (Hutchins 2011, p. 440). ${ }^{10}$ This allows us to cast the relation between local and global dynamics in terms of co-

\footnotetext{
${ }^{10}$ One might wonder about the following: even if one were to grant that the macroscale process is extended over spatial and temporal scales, does it follow that the dynamics of the component parts are also extended? To properly address this question we distinguish between two senses of 'extended'. First, if 'extended' means extended over spatial and temporal scales, then we can answer the question affirmatively. For example, in the Mexican wave, even the activities of the individual parts unfold over
} 
constitution - i.e., local and global dynamics jointly constitute one another. The notion of coconstitution is associated with the concept of reciprocal causation - a concept that lies at the heart of theorems in the physical sciences such as the slaving principle in physics (Haken 1983).

Table 1: Properties of synchronic and diachronic constitution

\begin{tabular}{|c|c|}
\hline Michelangelo's David & Mexican Wave \\
\hline- Synchronicity & - Diachronicity \\
\hline- Asymmetry & - Co-constitutive \\
\hline- Non-causal & - Reciprocal causation \\
\hline - Object-based & - Process-based \\
\hline
\end{tabular}

We now apply the constitutive properties of the Mexican wave to the infant/caretaker dyad, and show that the properties of the former map onto the properties of the latter. First, infant/caretaker interactions are inherently diachronic. Generally speaking, when two or more people interact, they tend to, on average and over time, modify their individual behaviour to those of others (Bernieri \& Rosenthal 1991). This not only impacts on social cognition but also facilitates social coordination (Barsalou et al. 2003). This ongoing attempt to modify or align behaviour is known as synchrony. Technically, synchrony is known as generalised synchrony, and refers to the matching of rhythmic behavior in chaotic dynamics, commonly in skew-product (i.e., master-slave) systems.

In the case of infant/caretaker dyads, generalised synchrony is applied to reciprocally coupled dynamical systems. Generalised synchrony has been extensively documented, most famously by Huygens (1673) and his work on pendulum clocks - matching their rhythms via the motions of the beam from which they were suspended (Friston \& Frith 2005). In infant/caretaker dyads, synchrony should not be associated with the synchronisation of two pendulums, as synchronous behaviour in the infant/caretaker case involves much more than mere 'rhythmic copying'. As Varga explains: "Synchrony, rather, refers to the co-creation of patterns that involve not mere copying, but also the temporally and dynamically variable completion of each other's vocalizations and gestures" (2015, p. 6). In this sense, the synchronous activity of non-verbal behaviors of the infant and the caretaker involves a degree of temporal coordination. It is for this reason that Varga (2015) says that the emotion regulation in infant/caretaker dyads is not an ability of a single individual but a socially extended process comprising both infant and caretaker. Against this claim, one might worry that reciprocal causation need not always imply co-constitution. For instance, you might think that the movements of two individuals are tightly or

temporal and spatial scales; yet the temporal scales over which the parts unfold are much faster than the temporal scales over which the whole unfolds. Second, if we take 'extended' to imply an extended socialcognitive process, then the Mexican wave is best understood as a social-cognitive extended process comprised by dynamics at the scale of the individual. 
reciprocally coupled during dancing, and yet still hold that the individuals in question remain ontologically distinct. We would not want to say that the ontology of the individuals is in question; rather, the point is that the activity of the two agents co-constitute one another in the sense of reciprocally causing the ongoing and temporally extended act of dancing. The same point, we submit, holds in the infant/caretaker example. ${ }^{11}$

Second, emotion regulation in infant-caretaker dyads exhibits the hallmark of jointly coupled dynamical systems; namely, self-organisation. This means that dyadic emotion regulation selfassembles not from a predetermined intention but spontaneously. Crucially, self-organised ensemble behaviour can be shown to have top-down effects on the individual constituents of the joint system. This follows from the slaving principle, which we described above. In this sense the macroscale process constrains - also known as enslaves - the activity of behaviour at the microscale. At the same time, of course, microscale behaviour generates the macroscale process of emotion regulation. The influence between these different scales of dynamics is mutual - there is not such a thing as a privileged - or relatively fundamental - scale of dynamics. This point is nicely expressed by Thompson, as he says: "At this dynamic [scale], the distinction between pre-existing parts and supervening whole has no clear application. One might as well say that the components ... emerge from the whole as much as the whole ... emerges from the components" (2007, p. 423). Hence, on the enactive account of social cognition, it makes no sense to privilege the parts (i.e., microscale processes) over the whole (i.e., ensemble behavior). On this view, parts and whole co-constitute each other. ${ }^{12}$

Table 2: Properties of synchronic and diachronic constitution, extended

\begin{tabular}{|c|c|c|}
\hline Michelangelo's David & Mexican Wave & Infant-caretaker dyad \\
\hline - Synchronicity & - Diachronicity & - Diachronicity \\
\hline - Asymmetry & - Co-constitutive & - Co-constitutive \\
\hline - Non-causal & - Reciprocal causation & - Reciprocal causation \\
\hline
\end{tabular}

\footnotetext{
${ }^{11}$ A different worry might be the following: even if there is a tightly coupled system, it could quite easily be the case that there is an asymmetry in the level of understanding relative to the comprising members of the system. No doubt there is something to this thought - as there might be some people that simply react to the Mexican wave as it is unfolding across the stadium, while other participants have a much greater grasp of the history and connotations of the event. The same can be said about the infant/caretaker dyad. Even so, we do not think this is a sufficient condition for rejecting our claim, as we are not saying that extended social cognitive processes can be extended if and only if there is symmetry in the level of understanding between the participants. In the infant/caretaker case we are rather focusing on the orchestrated and ongoing dynamics of emotion regulation.

${ }_{12}$ One worry might be that it is unclear how to settle the issue of what comprises the constitutive relation. In the context of this paper, especially the discussion over the extent of minds, it is not uncommon to invoke conditions such as non-derived content (Adams \& Aizawa 2001) or functional profile (Rupert 2009). There is however well-known problems with both conditions. For example, there is still no naturalised account of non-derived content, making it close to or identical with mere philosophical intuition. Although we do not explicitly address this issue here, our own bet on how to determine what makes up the constitutive relation is by appeal to either interventionism (Kirchhoff \& Meyer 2017; Meyer 2018) or mutual manipulation (Kaplan 2012; Kirchhoff 2017). The benefit of going down this manipulation route is that it does not rest on specific philosophical intuitions but on scientific practice.
} 


\begin{tabular}{|l|l|l|}
\hline - Object-based & - Process-based & - Process-based \\
\hline
\end{tabular}

This concludes our conceptualisation of diachronic constitution in the context of social cognition. To paraphrase van Gelder and Port (1995), "imposing the [properties of synchronic constitution] onto the [case of dyadic emotion regulation] is like wearing shoes on your hands. You can do it but gloves fit a whole lot better." (1995, p. 2)

\section{The Causal-Constitutive Fallacy (Fallacy)}

One immediate and difficult question that arises in response to the diachronic constitution claim concerns the widely acknowledged view that facts about causation do not entail any facts about constitution, given that these relations of dependence are considered to be metaphysically distinct (Bennett 2011). For instance, Carruthers (2015), following Block's (2005) review of Noë's (2004) enactivist account of perception, argues that enactivist explanations of social cognition "persistently conflate cause and constitution", and that at best enactive explanations establish that social "perceptual contents both give rise to, and are influenced by, sensorimotor knowledge" (Carruthers 2015, p. 499). For this reason, Carruthers maintains that enactive explanations do not "establish that [perceptual contents] are constituted by such [sensorimotor] knowledge" (p. 499). While Block and Carruthers specifically target enactivist claims regarding sensorimotor dependencies, the objection is a version of the coupling-constitutive fallacy (CCfallacy) (Adams \& Aizawa 2001, 2006, 2008). We consider the CC-fallacy, for if it holds the enactive framework will not get off the ground.

Targeting Noë's (2004) sensorimotor theory of perception, Block (2005) argues that Noë only shows "sensorimotor contingencies have an effect on experience, not that experience is even partially constituted by - or supervenes, constitutively on - bodily activity." (2005, pp. 4-5). Block's objection comes from what he describes as the orthodox view that presents the issue of the constitutive supervenience base for perception as what is minimally "a metaphysically necessary part of a metaphysically sufficient condition of perceptual experience" (2005, p. 5). Thus, according to the orthodox view, in order to determine what factors play a constitutive role in perceptual experience, we should determine what factors satisfy a minimal metaphysical sufficient condition for perceptual experience to occur.

In order to do this, Block claims that one needs to determine "the minimal supervenience base for an experience that occurs at time $t$ " as it will be "an instantiation of a physical property at $t$-according to the orthodox view" $(2005$, p. 6). By taking this synchronic approach to analysing the metaphysics of perception, Block argues that nothing outside of the brain is part of the minimal supervenience base for perceptual experience. This means that according to the orthodox view, only internal neural brain processes can satisfy the constitutive condition for perceptual experience. From this, Block (2005) emphatically concludes:

there is often a process of perception that involves bodily activity-one moves closer to get a better look—but that should not be conflated with the very different idea that perceiving is an activity or, worse, that perceptual experience is an activity. And even if perceptual experience depends causally or counterfactually on movement or another 
form of activity, it does not follow that perceptual experience constitutively involves movement (2005 p. 6).

The orthodox view arrives at this conclusion because all that matters to determine the content of the perceptual experience at time $t$ is the brain-bound neurological processes at time $t$. Hence, Block claims that even if we hold environmental variables as fixed, "only the features of the brain now are needed to determine the phenomenal character of experience now' (2005, p. 6). Thus, Block argues that Noë's claim that sensorimotor dependencies, which are the embodied and embedded dynamical relationships between the organism and its environment, constitute perceptual experience is fallacious, as the claim conflates causation and constitution.

Ascribing to the same orthodox view, Adams and Aizawa $(2001,2008)$ argue that causation and constitution are metaphysically distinct because the relation between cause and effect is temporal - causes precede their effects - whereas the constitution relation between parts and whole is cast as atemporal. Following on this, their version of the CC-fallacy argues that mere causal coupling of some internal process with a broader environment does not necessarily extend that process into the broader environment and conflates causation with constitution (Adams \& Aizawa 2001, p. 56). The claim is: "a process $P$ may actively interact with its environment, but this does not mean that P extends into its environment" (2001, p. 56). They argue that we cannot simply assume that causally coupling a process $X$ to a cognitive process $Y$ will be sufficient to make $X$ a constitutive component of the $Y$ cognitive process (2001, p. 93).

Adams and Aizawa anticipate an appeal to notions such as generalised synchrony and the dynamics of coupling or circular causation that we have presented. In order to show that the fallacy still applies to such a move, they (2008) examine the classic coupled pendulums example. They argue that even in cases of generalized synchrony, "the motions of the first pendulum are still motions of the first pendulum. The motions do not extend form the first pendulum into either the spring or the second pendulum" (p. 109). Just because the two pendulums are reciprocally coupled, they argue that this does not give us reason "to think that there is no such thing as the motions of the first pendulum" (109). Their argument is that just because the first pendulum is coupled to the second and its behaviour is modified by the second pendulum's behaviour, this does not demonstrate that the motion of the first pendulum extends into the spring and into the second pendulum. Thus, Adams and Aizawa ask why we should then think that a comparable modification of a cognitive process by being coupled to another external process should convince us that the external coupled process constitutes the cognitive process (p. 110). For this reason, the fallacy allegedly still applies to our diachronic account of constitution.

There is good reason to resist the feasibility of the CC-fallacy; namely Hurley's (2010) causal-constitutive error error argument. The argument claims that "explanations tend to be treated as causal or constitutive with no independent justification, in accord with prior assumptions or intuitions about boundaries, which often themselves have no clear basis and do not illuminate the distinction" (2010, p. 106). For example, Block's objection to Noë is that only brain-bound processes will fulfill the minimal supervenience base. But why think so? Adams and Aizawa state that only content instantiated in the head of individuals can serve as a mark of the mental, for only such content is in need of no further grounding in, say, social norms and cultural practices. There is however no agreed upon theory of non-derived content. So this is at best a 
bet on a future theory of non-derived content or a non-sequitur. Indeed, as Hurley (2010) observes, whether one determines enactivist constitutive claims to be fallacious or not, is going to turn "on some theoretical account of content, or phenomenal quality, or their enabling processes--but this is just what is at issue between internalism and externalism" (Hurley 2010, p. 106). ${ }^{13}$

\section{Beyond the Poverty of the Interactional Stimulus Argument}

No doubt there is something correct about Hurley's (2010) observation that the distinction between constitution and causation need not be co-extensive with any internal-constitutive and external-causal divide. Even so, it is still possible to argue that deflating the CC-fallacy is not a sufficient condition for thinking that enactive social cognition is correct. In this final section we consider a reason for thinking that enactive social cognition still comes up short. We do this by focusing on the role and characteristics of anticipation in cognition in general and in social cognition specifically.

Stepp and Turvey (2010) point out that anticipation is an essential component to cognition in general - and by extension, in accounts of social cognition (see also Bickhard 2016). This is the case both for cognitivist and non-cognitivist views of cognition, as anticipation is cast as constitutively involved in enabling an agent to respond flexibly to its dynamic and non-linear environment. Thus, answering the question of how to understand anticipatory behaviour will impact non-trivially on the discussion between cognitivism and enactivism.

The ability to anticipate the changing world seems to require rather sophisticated cognitive capacities, such as the ability to apply knowledge (and/or theorising) to sensory observations in order to arrive at a situationally appropriate response. The reason for this is that there is no oneto-one mapping between an agent's mental states and her observable behaviour because the same behavior might be manifested by different mental states (Carruthers 2015). Once one acknowledges that such knowledge is both neurally instantiated and what grounds flexible patterns of behaviour it is but a short step to acknowledging cognitivism. According to Stepp and Turvey (2010), for "those inclined to the traditional view of mediation of behavior by representations, anticipation would seem to be an especially 'representation- hungry' problem ... that is, one that cannot possibly be manifest without special internal states." $(2010$, p. 148$)$.

\footnotetext{
${ }^{13}$ As the determining factors regarding constitutive claims is just the issue at debate between cognitivists and enactivists, Hurley argues that the CC-fallacy is question begging, as it turns on the assumption, without any independent argument or empirical support, that the distinction between constitution and causation is such that only constitutive factors are internal to an organism and causal factors are external to organism (2010, p. 106). In order to avoid committing this error, Hurley recommends that we do not operate with prior assumptions regarding the causal-constitutive boundary (2010, p. 106). Hence, without an additional argument or further evidence to secure that the distinction between constitution and causation maps onto internal states being constitutive, while external states merely play a causal role in the instantiation of some specific phenomenon, the coupling-constitution fallacy cannot establish its conclusion without begging the question in its favor.
} 
On this cognitivist view, anticipation involves a system encoding or instantiating a model of itself and its local environment, enabling it to predict the causes of its sensory observations - at least approximately.

The cognitive neuroscientist Anil Seth frames anticipation along such cognitivist lines in understanding the architecture of cognition in general. He argues that enactive accounts of anticipation should be understood in terms of internal (i.e., brain-bound) hierarchical models of sensorimotor dependencies - viz., "counterfactual knowledge of relations between particular actions and the resulting sensations" (2015, p. 16). In this fashion, face-to-face social cognition would be a matter of each individual trying to predict or anticipate the relevant mental states, including emotional states, of the other conditioned on a model. Hence, all the 'real' cognitive work is realised in a brain-bound and neurally instantiated model of the other, where 'the other' takes the form of the environment or external states - the target of the predictions.

Carruthers' account of social cognition, framed in terms of Theory Theory, similarly holds that anticipatory processes should be understood as constitutively brain-bound. He argues that "even enactivism cannot obviate the need for tacit theory" $(2015$, p. 499). This is the "poverty of the interactional stimulus' argument against enactive social cognition. Carruthers claims that even if we grant that sensorimotor knowledge facilitates social cognition in interactions, as enactive accounts claim, we would still need to explain how any particular sensorimotor expectancy, or anticipation, can be generated by just the bare behavior of the other (p. 499). Pointing to Gallagher's (2001) claim that emotions and goals are directly expressed in bodily actions, and so can be directly perceived without tacit theory, Carruthers (2015, p. 499) argues that Gallagher's claim is implausible.

Enactivists (Froese \& Gallagher 2012; Fuchs \& De Jaegher 2009) typically claim that a core assumption of cognitivism is the unobservability of mental states. But this need not follow. Indeed, Carruthers (2015, p. 499) may be correct to point out that this assumption is unnecessary. Rather, cognitivist explanations may simply assume that "mental properties cannot be simply seen ... independently of concepts and acquired knowledge of the world" ( $p$. 499). Hence, there is for Carruthers a need for conceptual knowledge to inform bare perception of behaviour.

Carruthers argues that since "there are no one-to-one correspondences between mental states and behavior", enactive accounts "need to explain why someone will, in one context, anticipate one action, yet in a subtly different context, or with subtle differences in the other's behavior, will anticipate something else" (2015, pp. 499-500). Carruthers' argument is that enactive accounts need to explain contextually sensitive behavioural tendencies. However, according to Carruthers, explaining contextually sensitive behavioural tendencies necessarily requires a tacit theory of such contextually sensitive responses. Therefore, enactive explanations cannot avoid having to appeal to an underlying tacit theory in order to account for anticipatory processes in social cognition. Formulated this way, the poverty of the interactional stimulus problem allegedly shows that without appealing to tacit theory in their explanation of social cognition, proponents of enactive social cognition cannot give a satisfactory explanation.

It is however possible to resist these cognitivist depictions of anticipation and thereby defuse the poverty of the interactional stimulus objection. For it is possible to provide an explanation of anticipation in social cognition without appeal to an underlying tacit theory or knowledge-based model. By embracing a radical enactive alternative that casts perception as 
an exploratory activity, which extends over time, "there is no need to introduce 'knowledge' as a kind of bond that holds together various percepts in order to explain" anticipation of contextually sensitive behavioural tendencies (Hutto 2005, pp. 399-400). By casting perception this way, the simplified claim is that the sensorimotor dependencies of the various sensory modalities can account for the anticipation of the subtle differences in the other's behaviour in subtly different contexts. Sensorimotor dependencies, in conjunction with the particular features of the other as encountered in a situated physical and social context, account for both the perception and anticipation of the qualitative differences of the other's behaviour tendencies (Hutto 2005, p. 400).

Enactive accounts that reject an appeal to behaviour mediated by representational models conceive of anticipation as instantiated in ongoing sensorimotor couplings between an agent and its milieu, which includes, from time to time, engagement with other agents (De Jaegher et al. 2010). To see this, consider that any attempt to lesion or destroy the coupling relation effectively disrupts or terminates the anticipatory process. This much is evident from the stillface experiment discussed above. In the initial case, the infant and caretaker mutually create a coordinated state, in which their affective states are shared directly in their embodied and embedded behaviour (Fuchs \& De Jaegher, 2009, p. 479). This is what we referred to as generalised synchrony. General synchrony allows us to associate the process of emotion regulation as a process of the ensemble, inducing an overall state of stability and coordination. The still-face intervention, however, breaks the coordinated macroscale scale process, and what we see is a reduction in synchrony and thus of the ability to anticipate future states conditioned on action.

There is an important temporal aspect to anticipation that is analogous to the processual temporal dynamics we discussed in the Mexican wave example, and one we can exemplify by returning to the still-face experiment. The ongoing and synchronous engagement between the infant and caretaker constitutes and explains what happens when the generalised synchrony of nonverbal behaviours of the infant and caretaker break down (Varga 2015). The spillover effect of the infant continuing to avert its gaze and display distress is a product of the infant now anticipating another future breakdown in the engagement.

Close inspection reveals that the infant and caretaker are unlikely to exhibit a special form of synchrony; namely, identical synchrony, where there is a one-to-one mapping between the states of the two agents - a case of what we might call 'perfect anticipation'. The infant and caretaker dyad is not always in perfect synchronisation, as they typically only have matching affects $30 \%$ of the time (Fuchs \& De Jaegher, 2009, p. 479). This speaks to the pair having to continuously negotiate and renegotiate their interaction with one another. Indeed, it is the temporally unfolding process of maintaining affective coordination through synchronisation, desynchronisation and resynchronisation that drives the interaction forward. Hence, on this enactive construal of anticipation, it is through the embodied and dynamic process of mutual affective resonance that the infant and caretaker are able to get a dynamic grip on the affectivity of the other, and anticipate and respond accordingly to the perceived affectivity of the other, in the process of the interaction (Fuchs \& De Jaegher 2009, p. 479).

Emotion regulation in the infant/caretaker dyad is an example of a situated action. There is more to say about what orchestrates and constrains anticipation than just sensorimotor coupling - there is also a sociocultural dimension to anticipation. To unpack this we follow Hutchins 
(2011) in thinking of situated actions as cultural practices. Cultural practices are "the things that people do in interaction with one another" (p. 440). This means that the emotion regulation in the infant/caretaker dyad is itself a cultural practice. As Hutchins puts it: "Cultural practices include particular ways of seeing (or hearing, or feeling, or smelling, or tasting) the world. [They] are not cultural models traditionally construed as disembodied mental representations of knowledge. Rather, they are fully embodied skills. Cultural practices organize the action in situated action" (p. 441). The nice thing about considering anticipation in social cognition in relation to cultural practices is that it avoids biasing the notion of anticipation toward internal, brain-based models and foregrounds the idea that sensorimotor couplings are embedded within particular sociocultural setting, which constrains how individuals act and perceive their world.

This brings out a deep reason for why it is a mistake to think of anticipation in terms of strict cognitivism. The cognitivist grounds social cognition in anticipation and conceives of ongoing and recurrent interaction as merely causally influencing the brain-based anticipatory machinery. On our enactivist account however, anticipatory processes do not underlie the capacity for social cognition in interaction; instead, they are realised and grounded in recurrent interaction between individuals. In other words, anticipatory processes are constituted in the interactional dynamics between the interactors and their relationships to their shared world. The ability of the interactor to attend to, recognise and respond to the others' emotions and intentional behaviour rests on counterfactual relations between perception and action, but this ability is grounded in situated, ongoing engagement with other agents, over multiple spatial and temporal scales.

Another significant departure of our enactive view of social cognition to cognitivism is the following: the cognitivist focus on model mediated behaviour gives pride of place to dynamics at the microscale at any given moment in time. Our diachronic account of constitution however not only casts social cognitive processes extended temporal processes, it also implies that the temporally unfolding patterns of engagement over time is explanatorily prior to what is the case at any given moment in time. Hence, in the infant/caretaker dyad, mutual affective resonance is constituted by in a reciprocally coupled two-body system, which is informed by a history of engagement (see also Di Paolo et al. 2017; Fuchs \& De Jaegher 2009). One cannot therefore explain social cognitive activity without such an explanation presupposing a wholly dynamic and diachronic view of the metaphysics of social cognition.

\section{Conclusion}

In this paper, we focused on clarifying and extending an enactive alternative to the dominant cognitivist account of social cognition. We did this by first articulating and describing a diachronic notion constitution using the examples of the Mexican wave and infant/caretaker dyads. Our analysis of these examples showed that embodied engagement can play a constitutive, rather than just a causal, role in social cognition. This provides one substantial reason for considering enactive social cognition as a bona fide alternative and rival to the cognitivist framework.

We then considered two versions of the C-C fallacy (Adams \& Aizawa 2001, 2008; Block 2005 ) that has been put forth against enactive accounts of cognition. We deflated this objection by establishing that the fallacy assumes problematically that the distinction between constitution and causation is co-extensive with casting internal elements as constitutive and external 
elements as causal. Following Hurley (2010) we argued that this assumption begs the question against exactly what is being debated between traditional cognitivism and enactivism. Thus, the CC fallacy fails to block our enactive diachronic constitution claims.

Having argued that embodied and embedded elements of intersubjective engagements can play a constitutive role in social cognition; we turned to address Carruthers' (2015) poverty of the interactional stimulus objection. This objection argued that the anticipatory processes that facilitate social cognition in interactions need to be mediated by internally realized models, i.e., a tacit theory of mind. In the final part of the paper, we dissolved this objection in the context of the infant/caretaker dyad by arguing that it is possible and proper to cast anticipatory processes as orchestrated and maintained by the sensorimotor couplings between individuals in face-toface interactions. This demonstrates that our enactive account of social cognition is an alternative, rival account to the dominant cognitivist approach to social cognition.

\section{References}

Abramova, E. and Slors, M. (2018). Mechanistic explanation for enactive sociality.

Phenomenology and the Cognitive Sciences:1-24.

Adams, F. and Aizawa, K. (2001). The bounds of cognition. Philosophical Psychology 14 (1):4364.

Adams, F and Aizawa, K. (2006). "Defending the bounds of cognition". In The extended mind, Edited by: Menary, R. 45-48. Aldershot, England: Ashgate.

Adams, F. and Aizawa, K. (2008). The Bounds of Cognition. Wiley-Blackwell.

Aizawa, K. (2010). "The coupling-constitution fallacy revisited". Cognitive Systems Research, Volume 11, Issue 4, pp. 332-342.

Barsalou, L.W., Simmons, K.W., Barbey, A.K., and Wilson, C.D. (2003). Grounding conceptual knowledge in modality-specific systems. Trends in Cognitive Sciences, 7(2), 84-91.

Barrett, L. (2011). Beyond the Brain: How Body and Environment Shape Animal and Human Minds. Princeton University Press.

Bennett, K. (2011). Construction area (no hard hat required). Philosophical Studies, 154, 79104.

Bernieri, F., and Rosenthal, R. (1991). Interpersonal Coordination: Behavior Matching and Interactional Synchrony. In R.S. Feldman and B. Rime (Eds.), Fundamentals of Nonverbal Behavior (pp. 401-432). Cambridge: Cambridge University Press.

Bickhard, M. H. (2016). The Anticipatory Brain: Two Approaches. In V. C. Müller (Ed.) (2016). Fundamental Issues of Artificial Intelligence. (259-281). Switzerland: Springer.

Block, N. (2005). Action in Perception. Journal of Philosophy 102 (5):259-271.

Carruthers, P. (2015). Perceiving mental states. Consciousness and Cognition 36:498-507.

Clark, A. and Chalmers, D. J. (1998). The extended mind. Analysis 58 (1):7-19.

Clark, A. and Chalmers, D. J. (2010). The extended mind. In Richard Menary (ed.), The Extended Mind. MIT Press. pp. 27-42.

Colombetti, G. and Krueger, J. (2015). Scaffoldings of the affective mind. Philosophical Psychology 28 (8):1157-1176.

De Jaegher, H. (2018). The intersubjective turn. In A. Newen, L. de Bruin, and S. Gallagher (Eds.), Oxford Handbook of 4E Cognition. Oxford: Oxford University Press. 
De Jaegher, H., Di Paolo, E. and Gallagher, S. (2010). Can social interaction constitute social cognition? Trends in Cognitive Sciences 14 (10):441-447.

Di Paolo, E. (2009). Extended life. Topoi, 28, 9-21.

Di Paolo, E. and De Jaegher, H. (2017) Neither individualistic, nor interactionist. In Durt, C., Fuchs, T. and Tewes, C. (Eds.) Embodiment, enaction, and culture, MIT Press.

Di Paolo, E., Buhrmann, T. and X. Barandiaran (2017). Sensorimotor Life: an enactive proposal. Oxford Press: Oxford UK.

Dupre, J., and Nicholson, D. J. (2018). Everything Flows: Towards a Processual Philosophy of Biology. Oxford: Oxford University Press.

Froese, T. and Gallagher, S. (2012). Getting interaction theory (IT) together: Integrating developmental, phenomenological, enactive, and dynamical approaches to social interaction. Interaction Studies 13 (3):436-468.

Friston, K. (2013). Life as we know it. Journal of the Royal Society Interface 10: 20130475. http://dx.doi.org/10.1098/rsif.2013.0475

Friston, K. and Frith, C. (2015). A duet for one. Consciousness and Cognition. http://dx.doi.org/10.1016/j.concog.2014.12.003

Fuchs, T. and De Jaegher, H. (2009). Enactive intersubjectivity: Participatory sense-making and mutual incorporation. Phenomenology and the Cognitive Sciences 8 (4):465-486.

Gallagher, S. (2001). The practice of mind: Theory, simulation, or primary interaction? Journal of Consciousness Studies, 8, 83-107.

Gallagher, S. (2017). Enactivist Interventions: Rethinking the Mind. Oxford University Press.

Gallagher, S. (2018). New mechanisms and the enactivist concept of constitution. In M. P. Guta (ed.) The Metaphysics of Consciousness (207-220). London: Routlege.

Gallese, V. (2014). Bodily Selves in Relation: Embodied simulation as second-person perspective on intersubjectivity. Philosophical Transactions of the Royal Society, London. Series B Biological Sciences, 369 (1644), $20130177-20130177$.

Gopnik, A., and Meltzoff, A. N. (1996). Words, thoughts, and theories. Cambridge, MA: The MIT Press.

Haken, H. (1983). Synergetics: an Introduction. Nonequilibrium Phase Transition and Selforganisation in Physics, Chemistry and Biology. Berlin: Springer.

Herschbach, M. (2012). On the role of social interaction in social cognition: a mechanistic alternative to enactivism. Phenomenology and the Cognitive Sciences 11 (4):467-486.

Hurley, S. L. (2010). Varieties of externalism. In Richard Menary (ed.), The Extended Mind. MIT Press. pp. 101-153.

Hutchins, E. (2011). Enculturating the Supersized Mind. Philosophical Studies, 152, 437-446.

Hutto, D. D. (2005). Knowing what? Radical versus conservative enactivism. Phenomenology and the Cognitive Sciences 4 (4):389-405.

Hutto, D. D. and Myin, E. (2013). Radicalizing Enactivism: Basic Minds Without Content. Cambridge, MA: MIT Press.

Huygens, C. (1673). Horologium oscillatorium. France: Parisiis.

Kaplan, D. M. (2012). How to demarcate the boundaries of cognition. Biology and Philosophy 27 (4):545-570. 
Kirchhoff, M. D. (2015). Extended Cognition \& the Causal-Constitutive Fallacy: In Search for a Diachronic and Dynamical Conception of Constitution. Philosophy and Phenomenological Research 90 (2):320-360.

Kirchhoff, M. D. (2017). From mutual manipulation to cognitive extension: challenges and implications. Phenomenology and the Cognitive Sciences 16 (5):863-878.

Kirchhoff, M. D. and Meyer, R. (2017). Breaking explanatory boundaries: flexible borders and plastic minds. Phenomenology and the Cognitive Sciences:1-20.

Krueger, J. (2010). Extended cognition and the space of social interaction. Consciousness and Cognition, 20, 643-657.

Meyer, R. (2018). The Nonmechanistic Option: Defending Dynamical Explanation. British Journal for the Philosophy of Science: axy034.

Nagy, E., Pilling, K., Watt, R., Pal, A., and Orvos, H. (2017). Neonates' responses to repeated exposure to a still face. PLOS, https://doi.org/10.1371/journal.pone.0181688

Noë, Alva (2004). Action in Perception. MIT Press.

Noë, Alva (2009). Out of Our Heads: Why You Are Not Your Brain, and Other Lessons From the Biology of Consciousness. Hill \& Wang.

Overgaard, S. and Michael, J. (2015). The interactive turn in social cognition research: A critique. Philosophical Psychology 28 (2):160-183.

Rupert, R. D. (2009). Cognitive Systems and the Extended Mind. Oup Usa.

Seth, A. K. (2015) The cybernetic Bayesian brain: from interoceptive inference to sensorimotor contingencies. In: Metzinger, Thomas K and Windt, Jennifer M (eds.) Open MIND. MIND Group, Frankfurt am Main, pp. 1-24.

Schönherr, J. (2016). What's so Special About Interaction in Social Cognition? Review of Philosophy and Psychology 8 (2):181-198.

Schönherr, J. and Westra, E. (2017). Beyond 'Interaction': How to understand social effects on social cognition. British Journal for the Philosophy of Science:axx041.

Stepp, N. and Turvey, M. T. (2010). On Strong Anticipation. Cognitive Systems Research, $11(2), 148-164$.

Thompson, E. (2007). Mind in Life: Biology, Phenomenology, and the Sciences of Mind. Cambridge, MA: Harvard University Press.

Tronick, E., Als, H., Adamson, L., Wise, S., and Brazelton, T.B. (1978). The infant's response to entrapment between contradictory messages in face-to-face interaction. Journal of the American Academy of Child Psychiatry, 17, 1-13.

Van Gelder, T., and Port, R.F. (1995) Explorations in the dynamics of cognition. In T. van Gelder and R. Port (Eds.), Mind as Motion. Cambridge, MA: The MIT Press.

Varga, S. (2015). Interaction and Extended Cognition. Synthese 193 (8): 2469-2496

Wasserman, R. (2004). The constitution question. Nous 38(4), 693-710.

Wilson, R. A. (2004). Boundaries of the Mind: The Individual in the Fragile Sciences - Cognition. Cambridge University Press.

Wilson, R. A. (2007). A puzzle about material constitution and how to solve it: Enriching constitution views in metaphysics. Philosophers' Imprint, 7 (5), 1-20. 